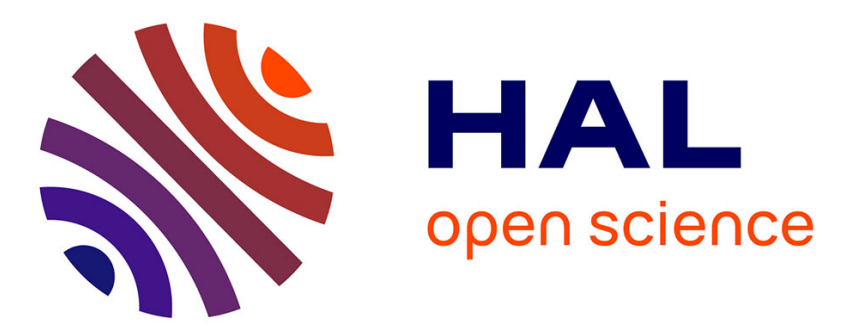

\title{
Des choix d'aménagement urbain porteurs d'inégalités sociales et environnementales: La gestion des déchets solides à Ouagadougou (Burkina Faso).
}

\author{
Issa Sory, Bernard Tallet
}

\section{- To cite this version:}

Issa Sory, Bernard Tallet. Des choix d'aménagement urbain porteurs d'inégalités sociales et environnementales: La gestion des déchets solides à Ouagadougou (Burkina Faso).. Flux - Cahiers scientifiques internationaux Réseaux et territoires, 2012, 3-4 (89-90), p. 79-89. halshs-01258483

\section{HAL Id: halshs-01258483 \\ https://shs.hal.science/halshs-01258483}

Submitted on 19 Jan 2016

HAL is a multi-disciplinary open access archive for the deposit and dissemination of scientific research documents, whether they are published or not. The documents may come from teaching and research institutions in France or abroad, or from public or private research centers.
L'archive ouverte pluridisciplinaire HAL, est destinée au dépôt et à la diffusion de documents scientifiques de niveau recherche, publiés ou non, émanant des établissements d'enseignement et de recherche français ou étrangers, des laboratoires publics ou privés. 


\section{DES CHOIX D'AMÉNAGEMENT URBAIN PORTEURS D'INÉGALITÉS SOCIALES ET ENVIRONNEMENTALES : LA GESTION DES DÉCHETS SOLIDES À OUAGADOUGOU (BURKINA FASO)}

Issa Sory, Bernard Tallet

Métropolis | « Flux »

2012/3 N 89-90 | pages 79 à 89

ISSN 1154-2721

Article disponible en ligne à l'adresse :

http://www.cairn.info/revue-flux-2012-3-page-79.htm

!Pour citer cet article :

Issa Sory, Bernard Tallet, « Des choix d'aménagement urbain porteurs d'inégalités sociales et environnementales : La gestion des déchets solides à Ouagadougou (Burkina Faso) », Flux 2012/3 (N 89-90), p. 79-89.

Distribution électronique Cairn.info pour Métropolis.

(C) Métropolis. Tous droits réservés pour tous pays.

La reproduction ou représentation de cet article, notamment par photocopie, n'est autorisée que dans les limites des conditions générales d'utilisation du site ou, le cas échéant, des conditions générales de la licence souscrite par votre établissement. Toute autre reproduction ou représentation, en tout ou partie, sous quelque forme et de quelque manière que ce soit, est interdite sauf accord préalable et écrit de l'éditeur, en dehors des cas prévus par la législation en vigueur en France. Il est précisé que son stockage dans une base de données est également interdit. 


\title{
Des choix d'aménagement urbain porteurs d'inégalités sociales et environnementales: La gestion des déchets solides à Ouagadougou (Burkina Faso)
}

\author{
Issa Sory \\ Bernard Tallet
}

\section{INTRODUCTION}

Lour es données disponibles à l'échelle de la ville de LOuagadougou ne permettent pas de rendre compte de la complexité des inégalités d'accès au service de collecte des déchets solides et d'exposition des citadins aux nuisances liées aux décharges. En effet, les enquêtes démographiques et sur les conditions de vie des ménages ne prennent pas en considération la fragmentation de la ville. Au contraire, ces études s'appuient sur des supports cartographiques - les zones de dénombrement - qui supposent que le tissu urbain est homogène. La réflexion ici proposée s'appuie sur des informations collectées entre 2008 et 2011 (1). Elle vise à analyser les inégalités environnementales liées aux déchets dans la ville de Ouagadougou, subdivisée en fonction de différences socio-économiques pouvant être saisies à travers les types d'habitat: la distinction reposant sur l'existence de cités, de zones loties et de quartiers irréguliers.

Les cités sont des zones loties qui ont la particularité d'être habitées par des couches sociales nanties (Jaglin, 1995; Ouattara, 1990; Marie, 1989). Environ 3\% des Ouagalais vivent dans ces cités. Les quartiers irréguliers, aussi appelés quartiers « spontanés » ou zones non loties, affichent certaines caractéristiques des bidonvilles dont $\mathrm{I}^{\prime}$ « absence de sécurité » foncière (Huchzermeyer, 2010, p. 216) et étaient habités, en 2006, par près d'un quart des Ouagalais (2). Le reste de la ville est peuplé de classes moyennes.
Toutefois la description du fonctionnement de la filière des déchets permet de décrypter les réalités de la fragmentation urbaine: I'implantation des infrastructures de collecte des déchets et le comportement des organismes précollecteurs dessinent, en effet, une ville " réelle » bien différente du découpage officiel en zones homogènes. De même, la répartition des décharges sauvages au sein de l'espace urbanisé met en lumière les contrastes quant à l'exposition des habitants aux nuisances liées aux déchets.

\section{LE SYSTÈME DE GESTION DES DÉCHETS ET LA FRAGMENTATION URBAINE DE OUAGADOUGOU}

Jusqu'en 2005, la collecte des déchets solides à Ouagadougou était essentiellement publique. Les précollecteurs, apparus suite aux défaillances puis à la liquidation de la structure publique de précollecte et de collecte des déchets (I'Office National des Services d'Entretien, de Nettoyage et d'Embellissement - ONASENE), dans un contexte de libéralisation entamée depuis 1990, déposaient les déchets dans des bacs à ordures. Disséminés dans la ville, ceux-ci étaient en majorité localisés dans I'arrondissement de Baskuy (arrondissement central regroupant 12 des 30 secteurs (3) de Ouagadougou). Les services municipaux étaient chargés de l'enlèvement des bacs à ordures pour déposer leur contenu dans les « décharges contrôlées » (4) situées à la périphérie de la ville (Jaglin, 1995). L'application du Schéma Directeur de Gestion des Déchets (SDGD), à partir de 2005, a 
introduit de profondes mutations dans la filière des déchets à Ouagadougou. Avec la nouvelle organisation, ces bacs ont été supprimés pour permettre l'émergence du secteur privé à toutes les étapes de la filière. Ils ont été remplacés par des centres de collecte dont l'implantation exige des espaces plus vastes capables d'accueillir plusieurs bacs - et accessibles aux moyens de transport.

\section{Nouvelle organisation de la filière des déchets: où implanter les centres de collecte?}

En 2005, les autorités municipales ont entrepris l'application du SDGD élaboré en 2000 sur financement de la Banque mondiale dans le cadre du troisième projet de développement urbain (1996-2004). Ainsi, la ville a été subdivisée en douze zones de collecte. Les associations communautaires - créées avec la libéralisation de la filière des déchets à partir de 1990 - ont été obligées de se regrouper en Groupement d'Intérêt Économique (GIE) pour postuler, avec les petites et moyennes entreprises (PME), à l'appel d'offres organisant l'attribution des concessions des zones de collecte. Les contrats de concession ont été signés en mai 2007 pour cinq ans. Cette forme d'organisation en GIE n'est pas propre à la ville de Ouagadougou. Dorier-Apprill (2002) note que le troisième Projet de Développement Urbain et Décentralisation - rédigé en 1995 - a suscité et soutenu la création de GIE à Mopti (Mali) pour la gestion de l'environnement urbain. Les services d'entretien de la ville ont été également concédés à ces nouveaux acteurs suite à un appel d'offres.

À Ouagadougou, les associations qui, pour diverses raisons (5), n'ont pas pu postuler à l'appel d'offres et qui n'ont pas de contrat de sous-traitance avec les acteurs formels (PME et GIE) constituent des acteurs informels. À ces derniers, s'ajoutent des charretiers individuels précollectant les déchets de façon permanente ou occasionnelle. Les charretiers individuels permanents et les associations informelles procèdent par des abonnements, comme les acteurs formels; tandis que les charretiers occasionnels pratiquent la précollecte comme une activité de substitution. Ces derniers sont rémunérés à l'acte. Cette rémunération peut être en espèces ou en nature. Le schéma recommande une redevance mensuelle de 1000 FCFA en moyenne par cour. Avec la concurrence entre les précollecteurs, la majorité des Ouagalais abonnés paie mensuellement 500 FCFA pour un " enlèvement » hebdomadaire. Les acteurs formels ont pour " devoir » de précollecter les ordures ménagères et d'éliminer les décharges sauvages de leur zone de collecte. Ces déchets précollectés doivent être déposés dans des centres intermédiaires (au nombre de 35) appelés centres de collecte. La mairie, à travers sa Direction de la Propreté, assure le transport des déchets des centres de collecte au Centre de Traitement de Valorisation des Déchets (CTVD) de trois arrondissements de la ville (Baskuy, Bogodogo et Boulmiougou), tandis que le transport des deux autres arrondissements (Nongr-Mâasom et de SigNonghin) et du secteur 8 est concédé à l'Entreprise Burkinabé des Travaux d'Équipement (EBTE). Cette entreprise est rémunérée par la mairie 4130 FCFA le mètre cube de déchets acheminés au CTVD (PSRDO-CER, 2010). À toutes les étapes de la filière, des tris sont effectués. Les objets triés (fer, aluminium, zinc, bidons, bouteilles, etc.) peuvent être recyclés ou vendus (6). La Direction de la Propreté a installé au CTVD deux cellules de traitement et de valorisation des déchets: une cellule de plastique pour le recyclage des déchets plastiques et une cellule de compostage des déchets biodégradables. En 2009, seulement la moitié des déchets produits ont été enfouis dans le CTVD (Direction de la Propreté, 2009; Boyer, 2010) (7).

L'organisation actuelle de la filière des déchets, tant en ce qui concerne les acteurs que les infrastructures, est nouvelle dans la ville si bien qu'il ne figure pas d'espace sur les plans de lotissement de Ouagadougou pour l'implantation des centres de collecte. Ainsi, l'une des premières difficultés à laquelle ont été confrontées les autorités municipales, dans le cadre de l'application du SDGD, a été de trouver des espaces pour leur localisation. Avant le schéma, les bacs qui recevaient les déchets des citadins occupaient des espaces réservés pour la construction d'infrastructures collectives (marché, jardin public, établissement scolaire, etc.). Avec le SDGD, il a fallu trouver des terrains pour la construction des centres de collecte. Interface entre les deux circuits majeurs de la filière (la précollecte et la collecte), les centres de collecte doivent être clôturés et comporter des bacs ainsi que le logement du gardien.

Par ailleurs, ces centres devaient être répartis de façon à réduire les distances à parcourir par les précollecteurs. Mais, dès lors que la ville a été construite, pendant près d'un demisiècle, sans inclure dans son aménagement la composante " déchets », où trouver ces espaces? Comment gérer l'implantation de ces infrastructures qui, facteurs de nuisances, peuvent être contestées par les riverains? Gouverner les exigences des citadins dans une ville aux espaces « finis » est devenu, avec le SDGD, le nouveau défi des autorités communales, dont les arbitrages obéissent à des logiques qu'il s'agit de déchiffrer. 


\section{Carte 1. Répartition spatiale des centres de collecte de Ouagadougou}

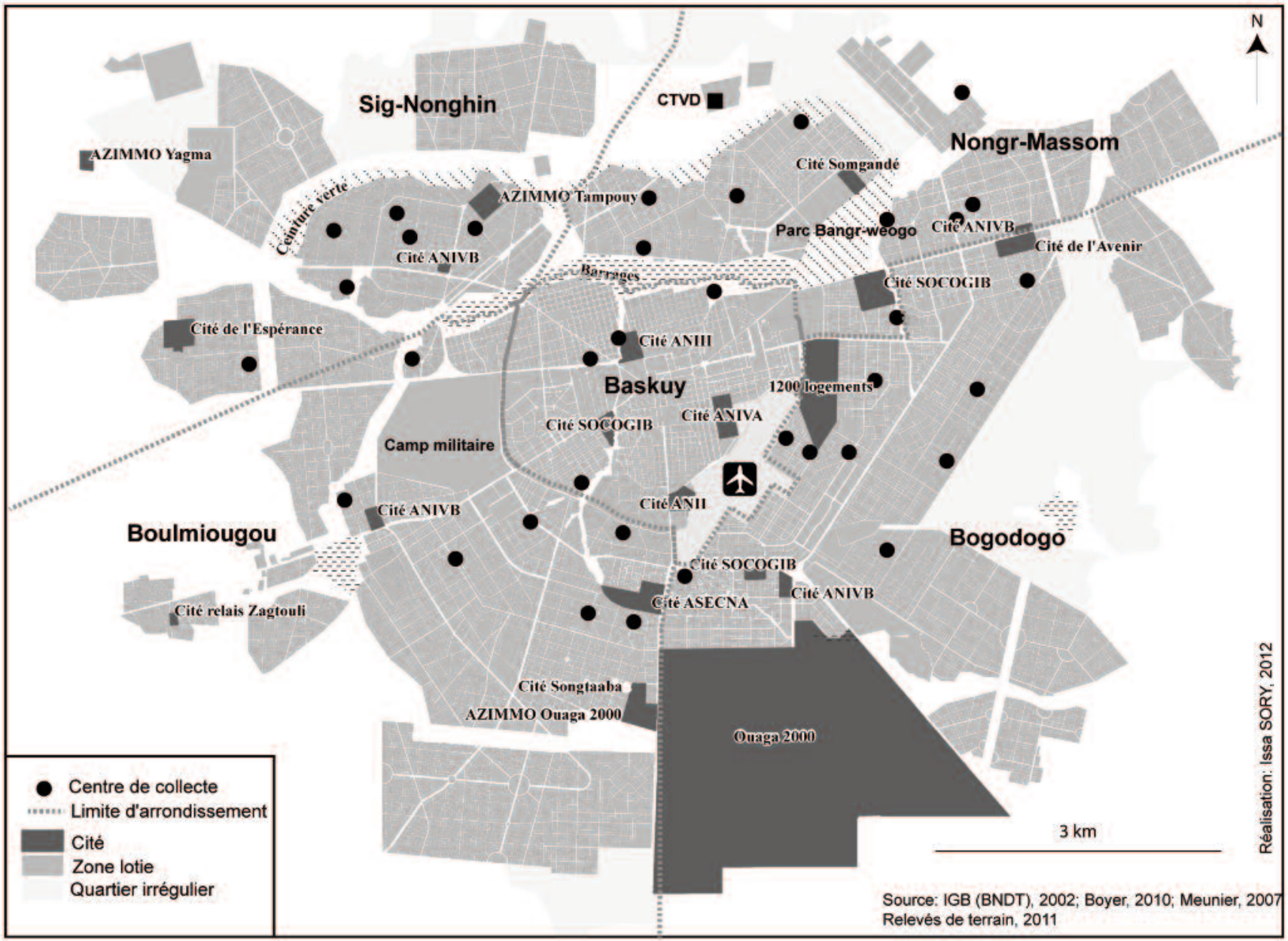

L'implantation d'un centre de collecte nécessite l'existence d'un espace vacant sur lequel peuvent être déposés des bacs et où peuvent être construits un local pour le gardien du site ainsi qu'un mur d'enceinte. Cet espace doit être desservi par des voies de communication praticables et suffisamment vastes " pour faciliter la manœuvre des camions " de collecte (Dessau-Soprin, 2000). Ainsi, des terrains réservés à la construction des infrastructures collectives ont été identifiés par les autorités municipales pour recevoir les déchets. Il s'agit, pour cellesci, de pallier les omissions des planificateurs par le détournement d'affectation des espaces vacants appartenant toujours à la mairie. Pour autant, la rareté d'espaces disponibles pour la construction de ces centres de collecte exclut de facto toute rigueur et rationalité dans leur répartition à travers la ville.

Dans ce contexte, le processus du choix d'un espace pour la construction d'un centre de collecte, selon la Direction de la Propreté, suit plusieurs étapes: I'identification de l'espace par les agents de la Direction de la Propreté, sa notification au maire central de la ville, la déclaration de l'espace comme centre de collecte, puis sa construction. Mais cette procédure ne semble pas s'appliquer à tous les espaces vacants de Ouagadougou. La répartition spatiale de ces centres révèle plusieurs visages de la ville et, notamment, la négation de la ville " invisible», à savoir les quartiers irréguliers et la volonté des autorités d'épargner aux cités les nuisances liées à ces implantations.

\section{Une inégale répartition des centres de collecte}

Les quartiers irréguliers et les cités ne disposent pas de centre de collecte; ceux-ci sont, en effet, tous localisés dans les zones loties, c'est-à-dire les quartiers de classes moyennes (cf. carte 1). 
Divers éléments expliquent la présence ou non de ces centres de collecte en fonction de la typologie des zones d'habitation, elle-même révélatrice du profil socio-économique des occupants. Ainsi, l'absence de centre de collecte dans les quartiers irréguliers souligne leur non-reconnaissance par les autorités municipales: en implanter reviendrait à reconnaître de fait ces quartiers déclarés «illégaux ». De même, l'implantation de centres de collecte uniquement dans les zones " régulières » démarque territorialement la ville « légale » de la ville «illégale». Par ailleurs, si l'absence de ces infrastructures dans les cités antérieures au SDGD peut s'expliquer par la rareté de terrains disponibles dans ces zones - toutes les parcelles étant construites et les espaces verts aménagés -, les nouvelles cités n'en disposent pas non plus. Seul le centre de collecte du secteur 3 se situe à environ 200 mètres d'une d'entre elles (Cité AN III).

Avec l'application du SDGD, les zones loties apparaissent comme les zones privilégiées d'implantation des centres de collecte. La proximité de ces centres pourrait être un avantage pour les riverains qui peuvent y déposer directement leurs déchets sans s'abonner, quand bien même cette pratique est proscrite.

Il apparaît, à travers l'implantation des centres de collecte, que les quartiers irréguliers sont marginalisés. Ces quartiers ne remplissent pas les critères pour être reconnus, leurs populations se trouvant dans l'illégalité foncière, et ils ne disposent d'aucune infrastructure conséquente. De plus, l'intégration de ces zones « illégales » dans la ville « légale » implique généralement la démolition de certains logements illégaux préexistants. L'absence de centres de collecte dans ces quartiers est ainsi guidée par le souci d'éviter leur démolition, au cas où ils ne répondraient pas aux impératifs d'un plan de lotissement futur. Enfin, l'absence d'infrastructures routières pour l'enlèvement des bacs exclut de fait les quartiers illégaux de l'identification des sites propices à la construction de centres de collecte par les autorités. À l'inverse, dans les cités, cette absence est voulue et souhaitée: les nuisances diverses des centres de collecte expliquent leur rejet par la frange de la population ouagalaise la plus fortunée. Ainsi, aucun espace n'est prévu pour en accueillir ni dans les cités construites après le schéma (Cité de I'Espérance) ni dans celles en construction (Ouaga 2000, cité de Yagma, etc.). Dès lors, le fonctionnement de ces territoires urbains privilégiés résulte de luttes d'influence auprès des autorités locales. Par exemple, le centre de collecte se trouvant à 200 mètres de la cité ANIII a été, dès son installation, menacé de délocalisation étant donné sa proximité avec la concession d'un ancien ministre. S'il s'y trouve toujours, cela s'expliquerait moins par la renonciation de cet homme influent que par son absence prolongée du pays (entretien avec M. Y, ex-responsable du Service Nettoyage et Collecte des Déchets, août 2008).

Ceci est une illustration des jeux de pouvoir qui s'opèrent dans le cadre de l'implantation des centres de collecte dans la ville de Ouagadougou. Les zones loties, abritant la quasi-totalité des centres de collecte de la ville de Ouagadougou, se retrouvent être les espaces d'application du SDGD; c'est ici que s'exerce effectivement le pouvoir des autorités communales, alors que les cités fonctionnent de fait avec une certaine autonomie, comme des quartiers fermés.

L'implantation des centres de collecte permet de délimiter des territoires urbains composés de marges (zones irrégulières), des zones qui échappent au contrôle des pouvoirs locaux (cités) et des espaces d'application du schéma (zones loties). Ces territoires déterminent aussi les comportements des précollecteurs du nouveau système de gestion des déchets solides à Ouagadougou.

\section{LA MISE EN OEUVRE DU SDGD: UN AMPLIFICATEUR DES INÉGALITÉS SOCIOÉCONOMIQUES}

Dans le cadre de l'application du SDGD, la ville a été subdivisée en douze zones de collecte attribuées aux GIE et PME ( $c f$. carte 2). Cette organisation, qui avait pour objectif d'uniformiser les modes de gestion des déchets (Meunier-Nikiéma, 2007), s'est très vite révélée inadaptée à la structuration du tissu urbain de Ouagadougou. De plus, la logique libérale instituée par le schéma a davantage complexifié les relations entre acteurs et accentué les inégalités d'accès au service de collecte.

\section{L'inadaptation du SDGD à la réalité urbaine}

Déjà en 1996, avec la liquidation de I'ONASENE, la concurrence entre précollecteurs s'était intensifiée - chacun intervenant à la fois dans plusieurs secteurs centraux, alors qu'aucun acteur ne précollectait dans des secteurs périphériques (Sory, 2009). Pour une répartition plus rationnelle des acteurs sur toute l'étendue de l'espace urbain, la ville a été divisée en douze zones de collecte, mais les critères de rentabilité des différentes zones de collecte, prévus dans le schéma, n'ont pas été suffisamment pris en compte (8), le découpage de la ville ayant 


\section{Carte 2. Les zones de collecte des déchets de Ouagadougou}

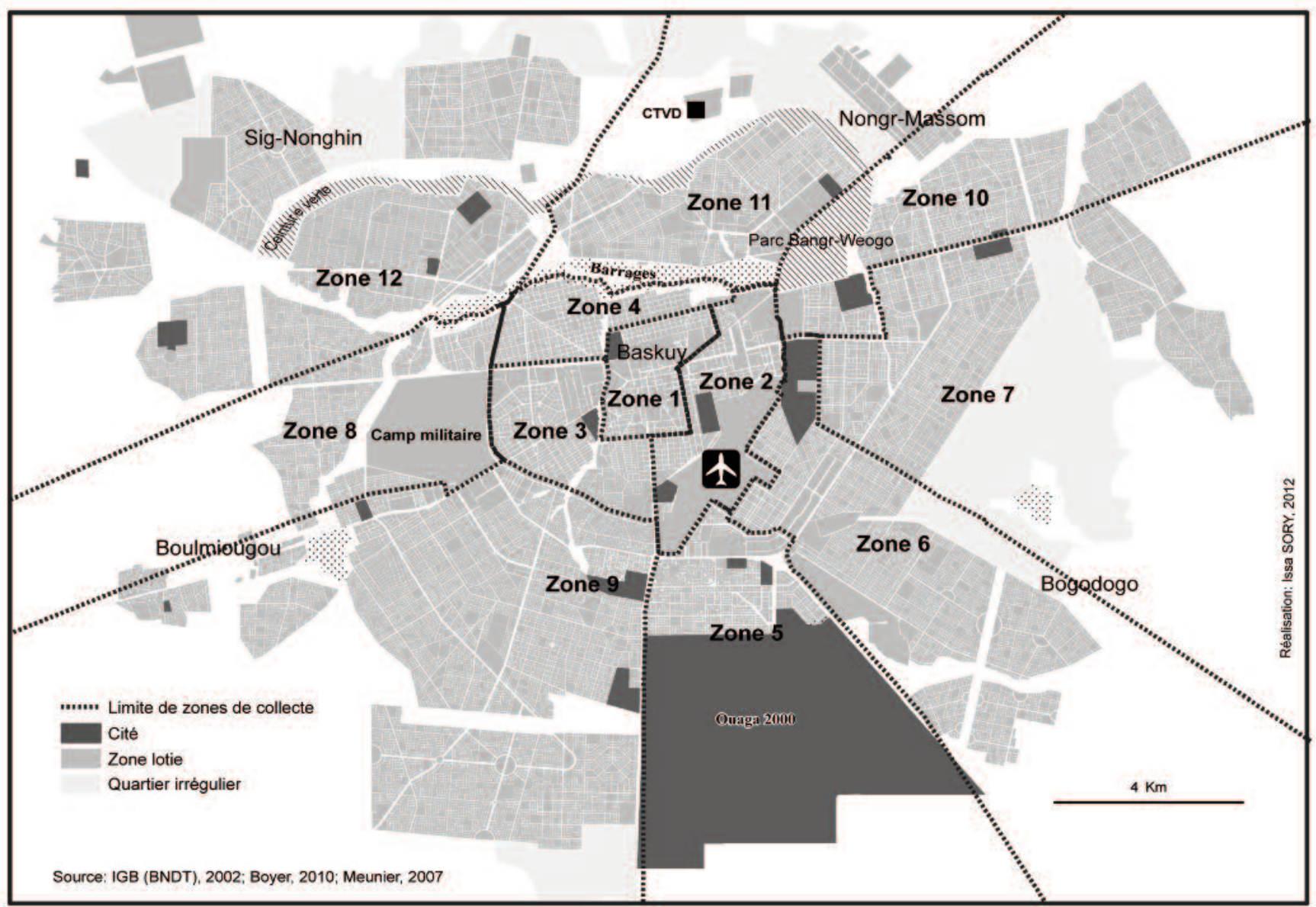

plutôt obéi à la perception que les autorités ont de la structuration du tissu urbain, selon trois ensembles bien distincts: la zone centrale (arrondissement de Baskuy), la couronne formée par les lotissements récents et les zones non loties (entretien avec Léon Paul Toe, ancien directeur des études et de la planification du ministère de l'Habitat et de l'Urbanisme et actuel directeur général de l'urbanisme et des travaux fonciers du même ministère, février 2010).

Pour Quénot (2010), les zones économiquement rentables pour les déchets seraient celles situées au centre-ville (arrondissement de Baskuy), tandis que les autres arrondissements (excepté Ouaga 2000) seraient peu rentables. Des zones de collecte comprenant ces deux types de quartiers - I'arrondissement central et la périphérie - devraient donc présenter de meilleures conditions de rentabilité économique, mais les pratiques réelles des collecteurs complexifient cette stratification binaire du tissu urbain (centre et périphérie); elles organisent une autre géographie économique des déchets à Ouagadougou.

L'appel d'offres pour l'attribution des zones de collecte a permis de retenir trois PME et neuf GIE. Ces nouveaux acteurs ont commencé à précollecter les déchets dans leurs « nouvelles zones » en 2007. En vue de les responsabiliser, ils ont le monopole de la précollecte des « ordures ménagères [...] et banales auprès des résidents, de même que de certains commerces, industries artisanales et institutions » dans leur zone respective et sont responsables de la propreté des espaces publics (Dessau-Soprin, 2000). Leur rémunération ainsi que le financement de toutes les étapes de la filière devant être supportés par les citadins - les "pollueurs »-, chaque Ouagalais devait $\mathrm{s}^{\prime}$ abonner et payer la redevance afin de permettre la gestion efficace et efficiente des déchets solides de la ville. Selon le sché- 
ma, cette redevance doit couvrir les frais de renouvellement du matériel de travail des concessionnaires de zone de collecte ainsi que leur bénéfice, le coût de transport des déchets des centres de collecte au CTVD et celui de leur enfouissement, ainsi que les royalties à payer à la mairie pour les services municipaux d'organisation de la filière. Au vu de l'importance de la redevance pour la structuration de la nouvelle filière, tous les moyens, y compris l'utilisation de la police municipale, devaient être mis en œuvre pour obliger les citadins à adhérer à ce modèle.

Cette délégation de service a très vite été débordée par les logiques des différents acteurs de la filière. En effet, si tous les précollecteurs attributaires de zones de collecte exerçaient déjà cette activité, certains n'ont pas pu postuler à l'appel d'offres et se sont donc constitués en acteurs informels. Il s'est alors établi une concurrence entre les différents acteurs (formels et informels), puis entre les GIE et PME (Tallet et al., 2011). Des enquêtes qualitatives auprès des acteurs de la filière, il ressort qu'aucun précollecteur n'intervient dans les quartiers irréguliers, du fait, selon eux, des difficultés d'accès à ces quartiers, de leur éloignement des centres de collecte et de l'insolvabilité des populations. Aussi, tous privilégient les cités.

Mme Zongo, responsable du GIE/ANGE'CO (entretien réalisé en février 2010), parlant des résidents de la cité de la Zone du Bois, indique ainsi que:

"Ce sont des gens qui sont en location. Tu ramasses aujourd'hui, demain la personne part et souvent de façon définitive. Mais pour ne pas perdre la cour, nous continuons à ramasser avec les gardiens même si personne n'y habite, jusqu'à ce qu'un autre locataire vienne. Dans cette cité, il y a des cours [cela correspond aux abonnés] de 1000 à 2000 Francs CFA par mois ».

Comparé à ce que ces précollecteurs pourraient gagner par mois avec les autres catégories d'abonnés (de 300 à 1000 FCFA), les cités sont plus rentables. Elles sont ainsi appelées les « bonnes zones » (9) de la ville.

Marginalisés dans le cadre de l'implantation des centres de collecte, les quartiers irréguliers sont donc aussi délaissés par les acteurs de la précollecte. En revanche, bien qu'ils soient réfractaires à l'implantation des centres de collecte dans les cités, les Ouagalais qui y habitent bénéficient malgré tout d'un service de précollecte. Ces discriminations positives et néga- tives, subies ou volontaires, sont également observables à travers la localisation des décharges dans la ville.

\section{Le débordement par les décharges sauvages: "le déménagement "}

La filière des déchets décrite dans le SDGD va des ménages au CTVD, avec les centres de collecte comme interfaces entre précollecte et collecte. Les adjudicataires des zones de collecte ont pour obligation de déposer les déchets précollectés dans les centres de collecte.

L'observation des pratiques des précollecteurs révèle que tous les types d'acteurs (informels et formels) déposent les déchets soit dans les centres de collecte, soit sur les décharges sauvages, ces dernières recevant également les déchets de certains citadins non abonnés. Les précollecteurs évoquent le retard de l'enlèvement des bacs des centres de collecte et/ou leur éloignement de certains quartiers pour déposer les déchets sur les décharges sauvages des zones de collecte.

"Souvent, les femmes [précollectrices] sont obligées de ramener les déchets à la maison parce que la Direction de la Propreté ne vient pas immédiatement lever les bacs des centres de collecte. Nous les déposons quelquefois au cimetière de Taptenga » (entretien avec Mme Zongo, responsable du GIE/ANGE'CO, février 2010).

Selon Mme Léocadie Bouda (CREPA, entretien réalisé en février 2010), cette pratique - appelée "le déménagement » (10) des déchets - sert d'arme dans le contexte de la concurrence. Les acteurs formels accusent ceux du secteur informel d'être responsables de la prolifération des décharges sauvages dans la ville. La répartition spatiale de celles-ci, créées par le « déménagement » des déchets, permet de dessiner d'autres territoires urbains et de révéler une autre réalité de Ouagadougou (cf. carte 3).

Selon la carte 3, plus de 95\% des décharges sauvages sont situées dans les zones loties. Elles sont majoritairement localisées dans la deuxième couronne de la ville, selon les étapes de la production de l'espace urbain. Il s'agit des quartiers nouvellement lotis des arrondissements périphériques (Bogodogo, Boulmiougou, Nongr-Mâssom et Sig-Nonghin). Seules deux décharges sont situées dans les quartiers irréguliers, tandis qu'il n'y en a aucune dans les cités.

Cette répartition permet de voir comment les Ouagalais 
Carte 3. Répartition spatiale des décharges sauvages (11) de Ouagadougou

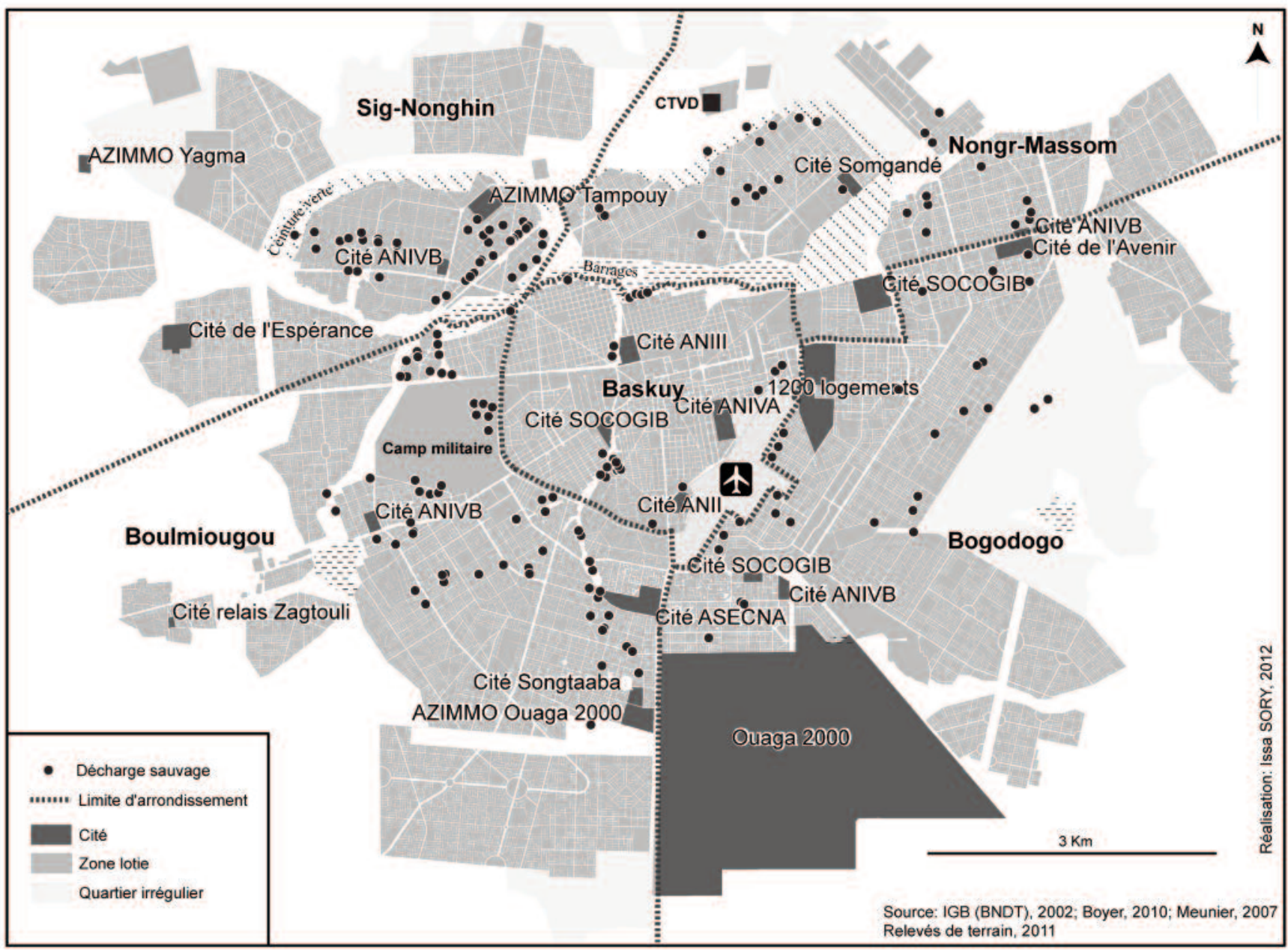

sont diversement exposés aux nuisances induites par les décharges sauvages selon leur zone d'habitation.

\section{L'IMPOSSIBLE ÉGALITÉ DEVANT L'ASSAINISSEMENT DE L'ENVIRONNEMENT}

Les infrastructures de collecte ont toutes été implantées dans la ville " légale », notamment dans les quartiers lotis créant une inégalité de fait avec les habitants des zones illégales, encore accentuée par les pratiques des précollecteurs qui n'interviennent que dans les quartiers lotis. La structuration de la filière des déchets à Ouagadougou et les interactions entre ses principaux acteurs tendent à réserver ce service aux plus nantis, remettant en cause le droit de tous à l'accès aux services de base.
Une offre de service surtout destinée aux plus aisés

La non-implantation des centres de collecte des déchets dans les cités, et donc l'éloignement de fait de ces infrastructures (par exemple, la zone résidentielle de Ouaga 2000), pourrait entrâ̂ner la présence de décharge. Or, aucune décharge sauvage n'existe dans cette partie de la ville, ses occupants y étant également hostiles.

L'enquête quantitative réalisée auprès des résidents de cent concessions des cités Songtaaba (Secteur 16) et AN IVB (secteur 22) a montré que $98 \%$ d'entre eux procèdent à un abonnement auprès des précollecteurs (informels et formels) pour l'élimination de leurs déchets ( $c f$. graphique). Cet important taux s'explique par la solvabilité de cette catégorie de la population, qui 


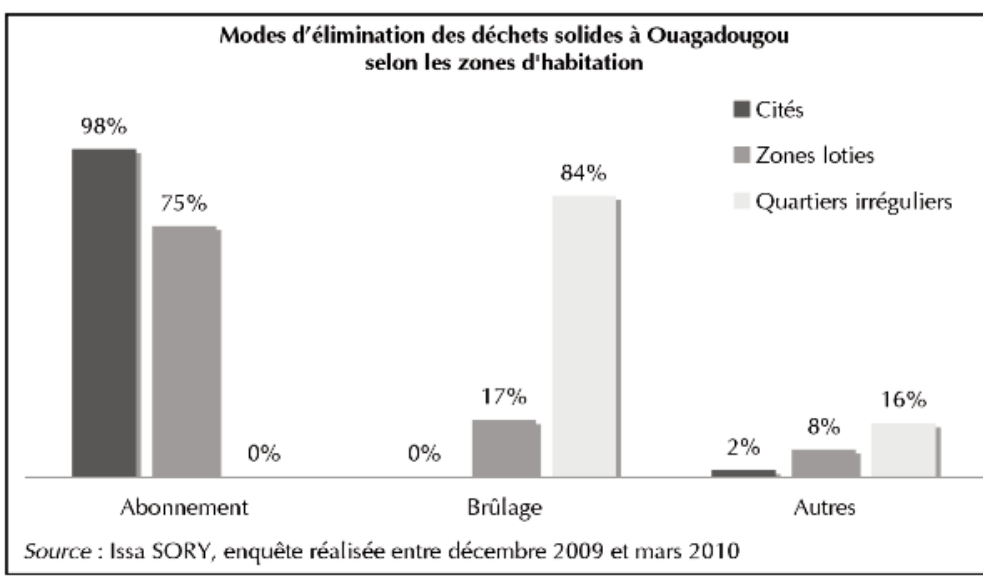

lui permet de payer la redevance. Le contenu des poubelles des cités motive aussi les précollecteurs. Ainsi, parlant du salaire des précollectrices de son association, M. Dieudonné Sawadogo (responsable de l'association Nabonswendé, entretien réalisé en avril 2011) souligne que:

"C'est vrai qu'elles ne sont pas bien payées mais elles disposent de clients non déclarés auprès de l'association. Elles vendent tout ce qu'elles gagnent [il s'agit des objets recyclables]. Dans la cité [cité Somgandé, secteur 25], elles gagnent beaucoup».

La concurrence à laquelle se livrent les précollecteurs pour le contrôle des « bonnes zones » est bénéfique pour leurs résidents, mais certains acteurs de la précollecte n'effectuent guère qu'un " déménagement » des déchets des cités vers les zones loties, déplaçant ainsi le problème et les nuisances.

L'accent mis sur les cités ne date pas d'aujourd'hui dans la capitale. En effet, à la fin des années 1980, l'établissement public à caractère administratif chargé de la collecte des déchets sur toute l'étendue du territoire - l'ONASENE - limitait ses actions, en plus des zones administratives et commerciales, aux quartiers d'habitants socialement aisés, notamment ceux de la Rotonde, Petit Paris, Zone du Bois, etc. (Jaglin, 1995). Pendant cette période, les dysfonctionnements du service public avaient justifié l'apparition du secteur privé. Le Centre de Gestion des Cités (CEGECI) (12), créé depuis 1987 pour gérer les cités construites à l'époque révolutionnaire, s'est ainsi attaché les services de ces opérateurs et a intégré la redevance de précollecte dans les loyers. Tous les résidents des cités bénéficiaient ainsi de ce service. Ce mécanisme d'accompagnement, en créant une certaine familiarité entre les précollecteurs et les locataires, facilite en outre l'abonnement de ces derniers lorsqu'ils deviennent propriétaires de leur logement.

\section{Les couches modestes les plus exposées aux nuisances}

Les centres de collecte de déchets sont localisés dans les zones loties où les acteurs de précollecte interviennent. Pourtant, c'est aussi dans ces zones qu'existe la presque totalité des décharges sauvages. Celles-ci sont localisées dans des ravins, parcelles sans constructions, cimetières, espaces réservés aux infrastructures collectives non encore aménagés, etc. L'existence d'espaces vacants dans ces zones peut expliquer la présence des décharges. En effet, les lotissements occasionnent des destructions de logements dans les zones antérieurement irrégulières. Cette " perte du capital foncier et immobilier » (Marie, 1989) nécessite généralement une phase de latence avant la reconstruction de logements sur les nouvelles parcelles "légales». Certaines d'entre elles sont vendues avec installation des propriétaires dans les quartiers illégaux pour « anticiper sur le lotissement ». Cette pratique se traduit par la succession de parcelles en attente d'être encore revendues ou mises en valeur. Dans ces zones ainsi que dans celles non loties, « la construction d'un logement définitif [...] s'étale sur une période de 10 ou 15 ans » (Prat, 1996).

Pour ce qui est des espaces réservés aux infrastructures collectives, ils restent longtemps sans mise en valeur: les routes se dessinent au fur et à mesure que les logements se construisent (Deverin-Kouanda, 1993); les espaces verts restent non aménagés, de telle sorte qu'ils sont souvent détournés de leur destination. Les autres infrastructures (sanitaires, scolaires, etc.) suivent difficilement le rythme du front d'urbanisation, si bien que celles des zones nouvellement loties sont implantées plusieurs années après les lotissements. Ces "dents creuses », du fait de l'insuffisance des centres - 35 centres pour une ville de plus de 36000 hectares - et de la concurrence à laquelle se livrent les précollecteurs, entraînent la constitution des décharges sauvages de la ville « légale ». Les campagnes d'élimination de ces décharges sont restées peu efficaces. En effet, selon le directeur de la Propreté, Sidi Mahamadou Cissé, " dans le cadre de la mise en œuvre du schéma directeur de gestion des déchets solides (débutée le 15 avril 2005), le Maire de la Commune de 
Ouagadougou, Simon Compaoré, a organisé une vaste campagne d'élimination de l'ensemble des dépotoirs 'sauvages' de la ville de Ouagadougou. [...] Malheureusement, quelques mois après, à la faveur de l'incivisme d'une certaine frange de la population, on a constaté la reconstitution ou la constitution de nouvelles décharges non contrôlées » (Le Progrès, repris par Lefaso.net, 13 septembre 2011).

Inversement, la densité du bâti et l'accent mis par les autorités municipales sur l'élimination des décharges dans l'arrondissement central (Baskuy) expliquent le peu de décharges sauvages dans cette zone.

Les enquêtes ont pourtant montré que $75 \%$ des résidents des zones loties sont abonnés auprès des acteurs de la précollecte pour l'élimination de leurs déchets ( $c f$. graphique). Ces données révèlent que l'accessibilité géographique (proximité des centres de collecte) et financière (paiement de la redevance) ne garantit pas forcément un environnement sain. En effet, ces décharges, résultat des jeux d'acteurs - le " déménagement » et le «transfert des risques » (Durand, 2010) des cités vers les zones loties -, en plus de leur répercussion sur le paysage urbain, polluent l'air, les sols et les eaux. Les rares caniveaux sont obstrués par les déchets des décharges sauvages. Elles constituent aussi des lieux de prolifération de germes et de vecteurs susceptibles de transmettre des maladies (Sory, 2009).

Les zones non loties, sans infrastructures de collecte, sont exclues de la filière par les acteurs de la précollecte qui « ne sont pas encore arrivés », selon les propos des résidents. Malgré cette marginalisation, il n'existe que deux décharges sauvages dans ces zones. À première vue, le niveau de vie modeste de la majorité des citadins des quartiers irréguliers - qui va de pair avec la faible production de déchets - pourrait être avancé comme argument pour justifier cette absence de décharges. Mais, I'analyse des modes d'élimination des déchets des résidents de ces zones montre qu'ils en brûlent une grande partie $84 \%$ (cf. graphique) -, avec toutes les conséquences environnementales et sanitaires que cela peut avoir en termes de pollution atmosphérique.

Le reste de leurs déchets est évacué dans d'anciennes carrières ou valorisé à travers la fabrication de compost par les habitants, à partir des fosses fumières familiales. Ces Ouagalais attendent d'être intégrés à la ville « légale » pour espérer bénéficier du service de la collecte des déchets.
Pourtant, tout laisse à penser que les quartiers irréguliers façonneront encore longtemps le tissu urbain de Ouagadougou. Même les « politiques volontaristes » du régime sankariste (1983-1987) n'ont pas pu empêcher durablement leur développement (Jaglin et al., 1992). Actuellement, plus qu'une voie de spéculation foncière ou de lieux d'habitation pour les néocitadins, la formation de quartiers irréguliers est le résultat de stratégies d'accession au logement « légal » (Boyer, 2010). En effet, dans un contexte d'inefficacité de la fonction de production de logement des différentes politiques urbaines et de la lenteur du processus de déconcentration et de décentralisation, la croissance démographique de la ville restera encore soutenue, avec pour conséquence la production de quartiers illégaux. De ce fait, ces quartiers constituent des " refuges » qui permettent de suppléer la crise du logement. Cependant, le SDGD laisse peu de perspectives à l'intégration des citadins qui y vivent. Les choix techniques et idéologiques du SDGD - des centres de collecte au lieu des bacs à ordures et la libéralisation de la filière des déchets - sont en contradiction avec l'inclusion des Ouagalais de ces quartiers dans la filière des déchets.

\section{CONCLUSION}

Les réformes en cours avec l'application du SDGD marginalisent les résidents des quartiers irréguliers, c'est-à-dire près d'un quart de la population ouagalaise. Les choix techniques opérés dans le cadre du schéma laissent peu de possibilités pour l'intégration de ces quartiers dans la filière des déchets. Les zones loties, correspondant aux quartiers formels des classes moyennes, territoires urbains d'exercice des pouvoirs locaux, constituent les seuls lieux d'implantation des centres de collecte des déchets. Ces infrastructures permettant de stocker les déchets issus de la précollecte, avant la phase de la collecte et de l'évacuation, sont primordiales puisqu'elles permettent un maillage du territoire et une efficacité de la gestion des déchets. Les résidents des cités, c'est-à-dire des quartiers aisés, sont quant à eux hostiles à l'implantation de ces centres du fait des nuisances qu'ils occasionnent.

Les logiques des précollecteurs ont conduit à l'amplification des inégalités d'accès au service de collecte des déchets et d'exposition à ses nuisances. Les acteurs formels - nés à la faveur de l'appel d'offres de 2003 - et informels ne précollectent que les déchets de la ville « légale ». Dans ce tissu urbain, ils procèdent $\mathrm{au}$ « déménagement » des déchets, dont I'une des consé- 
quences est la prolifération des décharges sauvages. La répartition spatiale de ces décharges montre qu'elles sont presque toutes localisées dans les zones loties. Il s'opère ainsi un « transfert des risques » liés aux déchets, des cités vers les quartiers lotis. Dans les quartiers irréguliers, I'élimination des déchets par le brûlage expose aux nuisances les Ouagalais qui y vivent. Ces inégalités - $d$ 'accès et d'exposition - apparaissent ainsi comme des marqueurs des inégalités environnementales dans la capitale burkinabé.

Par conséquent, la position d' « avant-garde » de « Ouaga la belle » est bâtie sur la gestion sélective de la ville: des cités assainies, des zones irrégulières exclues de la nouvelle filière et des quartiers lotis transformés en dépotoirs de la ville « légale». Fort de ce constat, la construction des nouvelles cités à la péri- phérie (cités AZIMMO de Ouaga 2000, Yagma, Tampouy) semble annoncer des recompositions dans les rapports classiques entre centre et périphérie. Cette dernière ne serait plus forcément cette partie de la ville dépourvue de services urbains...

Issa Sory est doctorant en géographie à l'Université Paris 1 PanthéonSorbonne sous la direction du Professeur Bernard Tallet. Sa thèse, qui s'inscrit dans l'axe "Questions urbaines dans les pays des Suds " de I'UMR 8585 PRODIG porte sur " la dynamique spatiale de l'habitat et la gestion des déchets solides à Ouagadougou (Burkina Faso)». soryssa@yahoo.fr

Bernard Tallet est professeur de géographie à I'Université Paris 1 Panthéon-Sorbonne. Au sein de I'UMR 8585 PRODIG, ses recherches portent sur les recompositions spatiales et les enjeux du développement (Burkina Faso, Cameroun, Mexique). Bernard.Tallet@univ-paris1.fr

\section{Notes}

(1) Les données utilisées dans cet article ont été collectées entre août 2008 et mai 2011 dans le cadre d'une thèse en cours. Les données quantitatives ont été collectées entre décembre 2009 et mars 2010 dans trois strates du tissu urbain de la ville: quartiers irréguliers (Simontoèga et Yagma), zones loties - centrales (secteurs 2, 3, 7, 8) et périphériques (16 et 21) - et cités (cité Songtaaba et cité AN IVB du secteur 21). Au total, 400 chefs de concessions ont été enquêtés dont 200 en zones loties et 100 dans les quartiers irréguliers et en cités. Des entretiens ont été réalisés auprès de tous les acteurs de la filière. Les centres de collecte et les décharges sauvages ont été localisés à I'aide d'un Global Positioning System (GPS) avec une marge d'erreur de 3 mètres.

(2) Le recensement général de la population et de l'habitation de 2006 a dénombré 320000 résidents dans les zones non loties de Ouagadougou, soit $22 \%$ de la population de la ville.

(3) Ouagadougou est subdivisée en 30 secteurs repartis dans 5 arrondissements (Baskuy, Bogodogo, Boulmiougou, Nongr-massom et Sig-nonghin).

(4) À cette période, quatre décharges, à peine qualifiables de décharges contrôlées, recevaient les déchets de la ville. Ces décharges étaient des sites légaux de dépôts des déchets solides collectés à Ouagadougou. Les déchets qui y étaient évacués ne subissaient aucune forme de traitement, ni régalage ou recouvrement (Jaglin, 1995, p. 183).

(5) Beaucoup de précollecteurs (associations et individuels) n'ont pas été informés du processus de concession de la précollecte. Certains, bien qu'ayant été informés, n'ont pu mobiliser les ressources humaines et financières pour le montage du dossier de l'appel d'offres; "le schéma [SDGD] a fait appel en fait, je peux dire, aux organisations les plus en vue » (entretien avec Mme Bouda Léocadie, CREPA, février 2010).

(6) Le kilogramme de fer est vendu 40 FCFA, celui d'aluminium entre 250 et 400 FCFA, celui de zinc 400 FCFA, de bidons de $50 \mathrm{cl}$ entre 5 et 10 FCFA et de bouteilles de $33 \mathrm{cl} 15$ FCFA (PSRDO-CER, CREA, 2010).

(7) Le calcul du taux de collecte est effectué à partir de la quantité des déchets produits (estimée à partir de l'effectif de la population - qui était de 2 millions selon l'enquête "Ouaga 2009 » coordonnée par Boyer et Delaunay - et de la quantité de déchets produits par chaque Ouagalais, soit 0,23 tonne) et de la quantité de déchets enfouis en 2009 (228000 tonnes). À partir de ces données, le taux de collecte était de $49,60 \%$ en 2009.

(8) Le SDGD propose la prise en compte du type de standing de l'habitat dans le découpage, afin que les différentes zones soient économiquement rentables pour les précollecteurs.

(9) Entretien avec M. Roger Tapsoba, responsable de I'entreprise ECONFA, février 2010.

(10) Pour elle, le « déménagement » consiste à transporter les déchets des sources de production aux décharges sauvages.

(11) Pendant la collecte des données, nous avons relevé que les décharges sauvages pouvaient remplir au moins trois bennes tasseuses de $12 \mathrm{~m}^{3}$. Certaines atteignent des dizaines de fois ce volume. L'estimation du volume et la localisation de ces décharges sauvages ont été effectuées avec l'aide des agents municipaux.

(12) Le CEGECI a pour objectif la perception du loyer des logements des cités qui sont actuellement en location-vente. Ce centre a été épaulé dans ses actions par la Société Nationale des Terrains Urbains (SONATUR) à partir de 1997. 


\section{BIBLIOGRAPHIE}

BOYER F., 2010, « Croissance urbaine, statut migratoire et choix résidentiels des Ouagalais: vers une insertion urbaine ségrégée? ", Revue Tiers-monde, n²01, pp. 47-64.

Dessau-Soprin, 2000, Schéma directeur de gestion des déchets dans la ville de Ouagadougou, Ouagadougou

DeVERIN-KouANDA Y., 1993, « De la fertilité rurale à la nuisance urbaine: les difficiles variations culturelles du Tampuuré (Tas d'ordures) en pays Mossi (Région de OuagadougouBurkina Faso) », Géographie et cultures, n6, pp. 121-134.

Direction de la Propreté, 2009, Rapport d'activités 2009, Ouagadougou

Dorier-Apprill E., 2002, "Gestion de l'environnement urbain et municipalisation en Afrique de l'Ouest: le cas de Mopti (Mali) », Autrepart, vol. 1, n²1, pp. 119-134.

Durand M., 2010, La Gestion des déchets et les inégalités environnementales et écologiques à Lima, entre vulnérabilité et durabilité, Thèse de doctorat, Université de Rennes 2

Huchzermeyer M., 2010, "Afrique: où et comment loger les urbains? ». In: Jacquet P., Regards sur la Terre 2010, Presses de Sciences Po, pp. 214-226.

JAGLIN S., 1995, Gestion urbaine partagée à Ouagadougou: pouvoirs et périphéries (1983-1991), Paris, Éditions Khartala-ORSTOM

Jaglin S., le Bris E., Marie A., Osmont A., Ouattara A., Ouédraogo J-P., Piron-Ayrand M., Poussi M., Sanou B., 1992, Les enjeux des extensions urbaines à Ouagadougou (Burkina Faso) 1984-1990, Compte rendu de recherche, Paris, ORSTOM

MARIE A., 1989, "Politique urbaine: une révolution au service de l'État », Politique Africaine, n³3, pp. 27-38.

MEUNIER-NikıÉMA A., 2007, "Géographie d'une ville à travers la gestion des déchets Ouagadougou », Mappemonde, n87. En ligne:

http://mappemonde.mgm.fr/num15/articles/art07305.pdf, consulté le 26 septembre 2012
Ouattara A., 1990, "Des lotissements aux cités: les facettes d'une politique de logement à Ouagadougou de 1960 à nos jours ». In: Le Bris E., Giannitrapani H., Maîtriser le développement urbain en Afrique sub-saharienne, Actes du colloque international de Ouagadougou, 1er-5 octobre 1990, ORSTOM, pp. 510-519.

PRAT A., 1996, «Ouagadougou, capitale sahélienne: croissance urbaine et enjeu foncier ", Mappemonde, $\mathrm{n}^{\circ} 1$, pp. 1824.

PSRDO-CER (Projet Stratégie de Réduction des Déchets de Ouagadougou-Création d'Emplois et de Revenus par des actions de collecte, de tri et de valorisation), 2010, Rapport de l'étude A.31: Réactualisation des données sur la problématique de la gestion des déchets dans la commune de Ouagadougou. En ligne: http://www.reduction-dechetsouaga.bf/etudes/cts/100322\%20A.3.1_du_18_mars_2010. pdf, consulté le 26 septembre 2012

PSRDO-CER, CREA (Cercle pour le Renforcement de l'Expertise en Afrique), 2010, Étude de base sur le rôle du secteur informel dans la gestion des déchets dans la commune de Ouagadougou, Rapport final

QuÉNOT H., 2010, La construction du champ politique local à Accra (Ghana) et Ouagadougou (Burkina Faso): le cas de la politique de gestion des déchets, Thèse de doctorat en Science politique, Université de Bordeaux IV, Institut d'Études Politiques de Bordeaux

SORY I., 2009, Gestion des déchets solides et risques sanitaires en milieu urbain, Mémoire de master, département de géographie, Université de Ouagadougou

Tallet B., Sory, I., Poze C., 2011, «Défi de l'expansion spatiale pour la gestion des services urbains: cas des déchets solides à Ouagadougou ", Conférence au 22e Festival International de Géographie, L'Afrique plurielle, paradoxes et ambitions 